\title{
Tolerancia, seguridad y eficacia de la preparación para colonoscopia con fosfato de sodio: el papel de la edad
}

\author{
D. Rodríguez-Alcalde, J. C. Marín-Gabriel, S. Rodríguez-Muñoz, J. de la Cruz Bértoloํㅜ, M. Barreales-Valbuena, \\ M. L. Manzano-Alonso y J. A. Solís-Herruzo
}

Servicios de Medicina del Aparato Digestivo y ${ }^{1}$ Epidemiología. Hospital Universitario 12 de Octubre. Madrid

\section{RESUMEN}

Objetivos: comparar la tolerancia subjetiva y los efectos adversos secundarios a la preparación para colonoscopia con dos productos, polietilenglicol (PEG) y fosfato de sodio (NaP), en pacientes adultos y en aquellos de 65 años o más.

Material y métodos: estudio retrospectivo apareado, tras escoger aleatoriamente a 140 pacientes de todos los que se sometieron a colonoscopia entre marzo de 2004 y mayo de 2005. Se investigó la presencia de los siguientes efectos adversos durante la preparación para la colonoscopia: fiebre, rectorragia, dolor abdominal, dolor perianal, náuseas, vómitos, sed, somnolencia, agitación, temblor y convulsiones. Se consideró mala tolerancia objetiva si el paciente presentó uno cualquiera de estos efectos. Asimismo, se recogió la tolerancia subjetiva al proceso de limpieza.

Resultados: se incluyeron 70 pacientes preparados con PEG y 70 pacientes con $\mathrm{NaP}(69$ mujeres y 71 hombres, con edad media de 60,6 \pm 14,8 años). No se demostró relación entre la tolerancia subjetiva o la presencia de efectos adversos y la limpieza con uno de los dos productos, ni en la población general ni en los pacientes de 65 años o más ( $p=0,09$ y $p=0,45$ en los ancianos, respectivamente). A pesar de ello, los pacientes preparados con $\mathrm{NaP}$ presentaron más náuseas que los tratados con PEG $(\mathrm{p}<$ 0,009 ), sobre todo las mujeres de 65 años o más. No se observaron efectos adversos graves en los pacientes preparados con $\mathrm{NaP}$. Los ancianos toleraron mejor cualquiera de las dos preparaciones mientras que las mujeres toleraron peor que los hombres la preparación, independientemente del producto empleado. La probabilidad de no completar la limpieza fue mayor en el grupo preparado con PEG y la calidad de ésta fue mejor en los que emplearon $\mathrm{NaP}$.

Conclusiones: la preparación previa a colonoscopia con $\mathrm{NaP}$ es tan bien tolerada, segura y eficaz como la realizada con PEG, también en pacientes ancianos sanos, a pesar de producir más náuseas. La limpieza obtenida con $\mathrm{NaP}$ es de mejor calidad.

Palabras clave: Fosfato de sodio. Polietilenglicol. Ancianos. Tolerancia.

\begin{abstract}
Objectives: to compare subjective tolerance and secondary adverse events to bowel cleansing prior to colonoscopy with polyethylene glycol (PEG) and sodium phosphate (NaP) in adult patients and in those 65 or more years old.

Material and methods: retrospective matched study, choosing 140 patients among all of those who underwent colonoscopy from March 2004 to May 2005. We investigated the presence of the next adverse events during bowel preparation: Fever, low digestive bleeding, abdominal pain, perianal pain, nausea, vomiting, thirst, somnolence, agitation, tremor and convulsions. We considered bad objective tolerance if the patient presented any one of these events. We also asked patients about subjective tolerance to preparation.

Results: seventy patients prepared with PEG and seventy with $\mathrm{NaP}$ were included (69 women and 71 men, mean age 60,6 $\pm 14,8$ years). There was no relationship between subjective tolerance or the presence of adverse events and bowel cleansing with any of the products in general population or in elderly patients ( $p$ $=0,09$ and $p=0,45$ in the elderly). However, patients prepared with $\mathrm{NaP}$ showed more nausea than those who employed PEG ( $\mathrm{p}$ $<0,009)$, overall women of 65 or more years old. There were no severe adverse events in patients prepared with $\mathrm{NaP}$. Elderly showed better tolerance than younger patients, and women worst tolerance than men, irrespective of the lavage preparation employed. Patients prepared with PEG unfinished bowel cleansing more frequently than those with $\mathrm{NaP}$. Cleanliness achieved with $\mathrm{NaP}$ was significantly better than that obtained with PEG.

Conclusions: bowel cleansing prior to colonoscopy with $\mathrm{NaP}$ is as well tolerated, safe and effective as with PEG, even in elderly healthy patients, although it causes more nausea. Cleanliness with $\mathrm{NaP}$ is better than that achieved with PEG.
\end{abstract}

Key words: Sodium phosphate. Polyethylene glycol. Elderly. Tolerance.

Rodríguez-Alcalde D, Marin-Gabriel JC, Rodríguez-Muñoz S, de la Cruz Bértolo J, Barreales-Valbuena M, Manzano-Alonso ML, SolísHerruzo JA. Tolerancia, seguridad y eficacia de la preparación para colonoscopia con fosfato de sodio: el papel de la edad. Rev Esp Enferm Dig 2008; 100: 17-23.

Recibido: $13-11-07$.
Aceptado: $19-11-07$.

Correspondencia: Daniel Rodríguez Alcalde. Servicio de Medicina del Aparato Digestivo. Hospital Universitario 12 de Octubre. Ctra. de Andalucía km 5,400. 28041 Madrid.e-mail: danielalcalde@hotmail.com 


\section{INTRODUCCIÓN}

La colonoscopia es actualmente la técnica de elección para el estudio del colon, siendo cada vez más empleada por ofrecer un alto rendimiento diagnóstico y permitir la realización de maniobras terapéuticas. Para la correcta visualización de la mucosa durante la prueba es necesaria una adecuada limpieza del colon (1-5).

El producto ideal para preparar el colon sería aquel que eliminase de forma rápida todo el material fecal en la totalidad de los pacientes, sin producir alteraciones en la mucosa ni efectos adversos, a un coste reducido (6). Hasta el momento no se ha desarrollado un preparado con estas características.

En los últimos años, el producto empleado mayoritariamente en la limpieza intestinal para la colonoscopia ha sido el polietilenglicol (PEG), un laxante osmótico no digerible y no absorbible. La composición por envase de PEG es $17,73 \mathrm{~g}$ de polvo con $15 \mathrm{~g}$ de polietilenglicol 4.000, 365,25 mg de cloruro sódico, 186,25 mg de cloruro potásico, $1408,5 \mathrm{mg}$ de sulfato sódico, $420 \mathrm{mg}$ de bicarbonato sódico y $120 \mathrm{mg}$ de bifosfato sódico. El PEG, debido a su iso-osmolaridad con el plasma y su neutralidad eléctrica, no produce absorción o excreción neta de agua o iones $(2,3,6)$, por lo que la administración de grandes volúmenes no causa cambios relevantes en el balance hidroelectrolítico del paciente. Sin embargo, la gran cantidad de líquidos que requiere esta preparación (habitualmente 4 litros) puede causar náuseas, vómitos y disconfort abdominal, que con cierta frecuencia motivan el incumplimiento terapéutico y la consiguiente preparación insuficiente del colon $(2,5-8)$.

Posteriormente se introdujo otro producto para la preparación del colon previa a la colonoscopia, el fosfato de sodio $(\mathrm{NaP})$. Se trata de un laxante salino compuesto por fosfato monosódico dihidratado y disódico dodecahidratado $(24,4$ y 10,8 g por cada $45 \mathrm{ml}$, respectivamente), que por su alto potencial osmótico actúa atrayendo plasma a la luz intestinal $(2,6,8,9)$. Una de las ventajas que ofrece es que requiere una menor ingesta de líquidos que la preparación clásica con PEG, por lo que se facilita el cumplimiento terapéutico. Sin embargo, debido a su composición y a la gran actividad osmótica que presenta, puede causar alteraciones hídricas e iónicas (hiperfosfatemia, hipernatremia, hipercloremia, hipopotasemia, hipocalcemia), a veces graves y con repercusión clínica (1,9-11). Por ello, está contraindicado en la insuficiencia cardiaca congestiva, la insuficiencia renal grave, la cirrosis hepática con ascitis, la enfermedad inflamatoria intestinal (EII) activa y en aquellos pacientes en los que la absorción del producto puede estar aumentada por megacolon, obstrucción intestinal o íleo. Pero, exceptuando estos casos, el $\mathrm{NaP}$ ha demostrado en diferentes estudios comparativos una eficacia y una seguridad iguales o mayores que el PEG, con menos de 1 caso de efectos adversos graves por millón de habitantes sanos y con una tolerancia al menos tan buena como este $(1-6,8,12,13)$. También en nuestro país, en un estudio multicéntrico, se han observado una eficacia y una seguridad similares del $\mathrm{NaP}$ con respecto al PEG, con una mejor tolerancia (14).

Estos trabajos han evaluado diversos factores que podrían determinar la tolerancia a ambas preparaciones, pero, según nuestro conocimiento, son muy pocos los que han estudiado específicamente la tolerancia y la seguridad en pacientes ancianos, considerando como tales a los de 65 años o más $(15,16)$. En estos dos estudios se ha comprobado que la limpieza con $\mathrm{NaP}$ es tan eficaz como con PEG y que es segura también en el grupo de mayor edad si se evita en los pacientes con contraindicaciones.

El objetivo del presente trabajo es comparar si la tolerancia subjetiva a ambos tipos de preparación es distinta y si es más frecuente la aparición de efectos adversos clínicos en los pacientes ancianos que llevan a cabo la limpieza intestinal con $\mathrm{NaP}$, para lo cual hemos realizado un estudio retrospectivo apareando por grupos de edad de 5 años a pacientes adultos y ancianos preparados con PEG y NaP, en relación de 1:1.

\section{MATERIAL Y MÉTODOS}

Los pacientes incluidos en el presente estudio han sido seleccionados de forma aleatoria entre todos aquellos que se han sometido a una colonoscopia, tanto electiva como urgente, en el Servicio de Medicina del Aparato Digestivo del Hospital Universitario 12 de Octubre (Madrid) entre marzo de 2004 y mayo de 2005.

Previamente a la realización de la prueba, todos los pacientes llevaron a cabo una preparación del colon que podía ser de dos formas: a) 16 sobres de PEG (Solución Evacuante Bohm ${ }^{\circledast}$; Laboratorios Bohm, Fuenlabrada, Madrid, España), 8 de ellos 48 horas antes de la colonoscopia, y los 8 restantes 24 horas antes, cada uno de ellos disuelto en 250 mililitros de agua; y b) dos tomas de $45 \mathrm{mi}-$ lilitros de NaP (Fosfosoda ${ }^{\oplus}$; Casen-Fleet, Utebo, Zaragoza, España) a las 13 y 21 horas del día previo si la exploración era por la mañana o a las 18 horas del día previo y las 9 horas del día de la prueba si esta era por la tarde. Cada toma de NaP debía ir disuelta en 200-250 mililitros de agua y el paciente debía consumir aproximadamente dos litros de líquido en total el día previo a la exploración. En ambas preparaciones los pacientes realizaron una dieta líquida clara las 24 horas previas a la colonoscopia y se administraron los enemas que precisaron hasta que los restos obtenidos fueron limpios.

Todos los pacientes eran mayores de edad y en todos los casos se obtuvo el consentimiento informado para la realización de la colonoscopia.

Las colonoscopias fueron llevadas a cabo por médicos adjuntos o por médicos residentes supervisados por un médico adjunto. El endoscopista valoró la limpieza del colon en el momento de la exploración como "buena" (menos del 20\% de la mucosa cubierta por restos líquidos 
aspirables), "regular" (del 20 al 50\% de la mucosa oculta por restos líquidos aspirables), "mala" (más del 50\% de la mucosa cubierta por restos líquidos o semisólidos) o "pésima" (exploración invalorable por restos sólidos). Se consideró como "adecuada" una preparación buena y como "deficiente" una limpieza regular, mala o pésima.

Para comparar la tolerancia "objetiva" entre PEG y $\mathrm{NaP}$ se recogieron las siguientes variables: fiebre, rectorragia, dolor abdominal, dolor perianal, náuseas, vómitos, sed, somnolencia, agitación, temblor y convulsiones durante la preparación. Se consideró "mala tolerancia objetiva" la presencia de uno cualquiera de estos efectos adversos. Asimismo, se preguntó a los pacientes por la tolerancia subjetiva a lo largo del proceso, que podía ser buena, regular, mala o pésima. La recogida de datos, una vez realizada la colonoscopia, se realizó por medio de entrevista personal o telefónica, dentro de un plazo máximo de 1 mes. Se excluyó a aquellos pacientes que no ofrecieron su colaboración o con los que no fue posible contactar.

Para el análisis estadístico se han utilizado los programas SPSS 11.0 para Windows y SAS. En la comparación de proporciones se ha empleado el test de Chi cuadrado o la prueba exacta de Fisher cuando fue necesario, mientras que en el caso de variables continuas se utilizó el test t de Student. Asimismo, se ha realizado un ajuste multivariable por medio de una regresión logística. Se estimó la fuerza de las distintas asociaciones por medio de odds ratio y su intervalo de confianza al $95 \%$. Se consideró significativo un valor de " $p$ " menor de 0,05 .

\section{RESULTADOS}

Se incluyeron en el estudio 140 pacientes: 70 preparados con PEG y 70 con NaP. De ellos, 69 (49,3\%) eran mujeres, siendo la edad media de los pacientes de 60,6 $\pm 14,8$ años, con un rango de 18 a 84 años. Se dividió cada grupo (PEG y $\mathrm{NaP}$ ) en dos subgrupos en función de la edad, uno formado por pacientes de hasta 64 años (38 preparados con PEG y 38 con $\mathrm{NaP}$ ) y otro por aquellos de 65 años en adelante (32 pacientes por subgrupo). Entre los pacientes preparados con PEG la edad media era de 60,7 $\pm 14,9$ (IC95\%: 57,2$64,3)$ y la mediana era de 63 años, mientras que la edad media de los pacientes preparados con $\mathrm{NaP}$ era de $60,5 \pm 14,8$ (IC95\%: 57-64) con una mediana de 62 años (Tabla I).

Con respecto a la distribución por sexos, se observó un desequilibrio en el grupo del $\mathrm{NaP}$ (41 varones frente a 29 mujeres), que no llegaba a ser estadísticamente significativo $(\mathrm{p}=0,06)$ (Tabla I).

De los 140 pacientes estudiados, 111 no presentaban comorbilidad relevante asociada, 17 sufrían cardiopatía, 12 neumopatía y 5 hepatopatía. Las indicaciones más frecuentes para realizar la colonoscopia fueron el seguimiento de pólipos (24 casos en cada grupo), la rectorragia (13 en el grupo del PEG y 22 en el grupo del NaP), la sospecha de neoplasia (PEG: 16; NaP: 4) y el estreñimiento (PEG: 7; NaP: 11) (Tabla I).
Tabla I. Características demográficas (un paciente puede presentar más de una indicación para la prueba)

\begin{tabular}{|c|c|c|c|c|}
\hline Caracteristicas de pacientes & \multicolumn{2}{|c|}{ PEG } & \multicolumn{2}{|c|}{$\mathrm{NaP}$} \\
\hline $\begin{array}{l}\text { Edad media } \pm D E \\
\text { Hombres/mujeres }\end{array}$ & \multicolumn{2}{|c|}{$\begin{array}{c}60,7 \pm 14,9 \\
30 / 40\end{array}$} & \multicolumn{2}{|c|}{$\begin{array}{c}60,5 \pm 14,8 \\
41 / 29\end{array}$} \\
\hline & $<65$ años & $\geq 65$ años & $<65$ años & $\geq 65$ años \\
\hline $\begin{array}{l}\text { Número } \\
\text { Indicaciones }\end{array}$ & 38 & 32 & 38 & 32 \\
\hline Pólipos & 12 & 10 & 10 & 12 \\
\hline Rectorragia & 5 & 8 & 15 & 7 \\
\hline Sospecha neoplasia & 7 & 9 & 1 & 3 \\
\hline Estreñimiento & 5 & 2 & 3 & 8 \\
\hline Otros & 17 & 9 & 19 & 9 \\
\hline
\end{tabular}

DE: desviación estándar.

Desde el punto de vista diagnóstico no se objetivó patología orgánica colónica en $43(31 \%)$ de los pacientes, presentando los $97(69 \%)$ restantes una o varias de las siguientes patologías: pólipos (42\%), divertículos $(30 \%)$, EII (5\%), neoplasia (4\%) y cambios inflamatorios inespecíficos (9\%) (Tabla II).

Tabla II. Diagnósticos endoscópicos con cada uno de los preparados (un paciente puede presentar más de uno)

\begin{tabular}{lcccc}
\hline Diagnóstico & \multicolumn{2}{c}{ PEG } & \multicolumn{2}{c}{ NaP } \\
\hline & $<65$ años & $\geq 65$ años & $<65$ años & $\geq 65$ años \\
Normal & 12 & 13 & 14 & 4 \\
Pólipos & 16 & 7 & 18 & 18 \\
Divertículos & 7 & 12 & 6 & 17 \\
Neoplasia & 2 & 1 & 0 & 3 \\
Ell & 3 & 0 & 3 & 1 \\
Otros & 5 & 2 & 3 & 3 \\
Total & 45 & 35 & 44 & 46 \\
\hline
\end{tabular}

Ell: enfermedad inflamatoria intestinal.

\section{Efectos adversos y tolerancia subjetiva}

En el análisis por separado de los efectos adversos no se constataron diferencias significativas entre ambos productos salvo en la aparición de náuseas, que presentó el 19\% de los pacientes preparados con PEG frente al $39 \%$ de los que lo hicieron con $\mathrm{NaP}(\mathrm{p}<0,009)$. Estratificando por edad, no encontramos diferencias en la presencia de náuseas en el grupo de hasta 64 años, pero sí una asociación entre estas y el NaP en los mayores de esa edad (PEG: 6\%; NaP: 28\%; $\mathrm{p}$ $=0,02)$. En cambio, estratificando por sexo, las mujeres presentaron más náuseas con NaP que con PEG (59 vs. $28 \% ; \mathrm{p}=0,009)$, mientras que en los hombres no se demostró tal asociación (24vs. 7\%; p = 0,06).

En el análisis conjunto no se apreciaron diferencias, ya que presentó al menos uno de dichos efectos adversos el $59 \%$ de los pacientes preparados con PEG y el $66 \%$ de los que recibieron NaP. Tampoco se observó relación entre uno de los dos productos y una mayor presencia de efectos adversos, considerados de forma global, tras estratificar a la población en los dos grupos etarios mencio- 
nados (en ancianos, PEG: 41\%; NaP: 50\%; $p=0,45$ ). Desde el punto de vista del sexo, aunque las mujeres presentaron un mayor número total de efectos adversos con $\mathrm{NaP}, 65$ en 25 de las mujeres preparadas con NaP frente a 53 en 26 de las que emplearon PEG ( $p=0,01)$, no apreciamos asociación entre una mala tolerancia objetiva y la preparación del colon con dicho producto (PEG: 65\%; NaP: $86 \% ; p=0,06)$. En los hombres tampoco se apreciaron diferencias entre ambos preparados.

Independientemente del producto utilizado, los pacientes de hasta 64 años toleraron objetivamente peor la preparación: $76 \%$ con al menos 1 efecto adverso frente al $45 \%$ de los pacientes de 65 años o más $(\mathrm{p}=0,0002)$, con una OR de 0,26 (IC95\%: 0,12-0,53) favorable a estos últimos. Asimismo, las mujeres presentaron peor tolerancia global objetiva que los hombres (74 vs. 51\%; $\mathrm{p}<0,005)$, con una mayor frecuencia de dolor abdominal $(\mathrm{p}=0,001)$, dolor perianal $(\mathrm{p}=0,001)$ y náuseas $(\mathrm{p}<0,002)$.

Al comparar la tolerancia subjetiva de los pacientes a la preparación no se observaron diferencias entre PEG y $\mathrm{NaP}(\mathrm{p}=0,22)$. Estratificando tanto por edad $(\mathrm{p}=0,09$ en ancianos) como por sexo tampoco se apreciaron diferencias entre ambos productos.

Sin embargo, si no tenemos en cuenta el producto utilizado, las mujeres presentaron peor tolerancia subjetiva $(\mathrm{p}=0,007)$, refiriendo un $52 \%$ de ellas una tolerancia regular, mala o pésima frente a un $34 \%$ de los hombres. A su vez, sólo el $30 \%$ de los pacientes ancianos presentó una tolerancia regular, mala o pésima frente al $54 \%$ de los pacientes de hasta 64 años ( $\mathrm{p}<0,004)$.

Además del sexo y la edad, se analizaron otros factores que pudieran ser relevantes en la tolerancia objetiva y subjetiva a la preparación. En el análisis univariado (e independientemente de si se empleó PEG o NaP) no se demostró asociación entre la presencia de comorbilidad y la aparición de al menos un efecto adverso o una peor tolerancia subjetiva. Tampoco se observaron diferencias de tolerancia objetiva o subjetiva en relación con la presencia o ausencia de patología orgánica en la colonoscopia. Estratificando en función del producto de limpieza utilizado no se apreció asociación entre cualquiera de las dos variables y la tolerancia, tanto objetiva como subjetiva.

En el análisis multivariable se observó que sólo la edad (OR: 3,7; IC95\%: 1,7-7,8) y el sexo (OR: 2,6; IC95\%: 1,2-5,6) influía en la presentación de uno o más efectos adversos durante la preparación, mientras que no se demostró una asociación de la comorbilidad o el diagnóstico endoscópico con dichos efectos. Con respecto a la tolerancia subjetiva los resultados fueron similares: el sexo (OR: 2,1; IC95\%: 1,02-4,2) y la edad (OR: 3; 1,4$6,4)$ fueron predictores de mala tolerancia.

\section{Limpieza}

La limpieza del colon fue significativamente mejor con $\mathrm{NaP}$ que con PEG ( $\mathrm{p}=0,007)$, obteniendo una preparación adecuada en el $66 \%$ de los pacientes que emplearon $\mathrm{NaP}$ frente al $47 \%$ de los que tomaron PEG $(p<0,03)$.

Los pacientes que se prepararon con PEG emplearon una media de 2,5 $\pm 2,1$ enemas, frente a los 1,7 $\pm 2,1$ de aquellos que lo hicieron con $\mathrm{NaP}$, sin que esta diferencia sea estadísticamente significativa.

Entre aquellos pacientes que no completaron la preparación, no se observaron diferencias en el grado de limpieza entre ambos productos, como tampoco entre los que la completaron, aunque en este caso hay una tendencia hacia una mejor preparación con $\mathrm{NaP}(\mathrm{p}=0,06)$.

\section{Preparación completada}

Entre los pacientes preparados con PEG, el 69\% completaron la pauta, mientras que en el caso del $\mathrm{NaP}$ finalizaron la preparación el $90 \%$ de los pacientes ( $p<0,002)$, obteniendo que la probabilidad de no completar la preparación era mayor con PEG, con una OR de 4 (IC95\%: 1,6-10).

Estratificando por edad, esta asociación se debía a los pacientes de 65 años o más (63 vs. 97\%; $\mathrm{p}=0,0006$ ), ya que en aquellos menores no se apreciaron diferencias. Por otro lado, si se estratifica por sexo, se observa que las mujeres cumplieron peor la pauta con PEG (63 vs. 93\%; p < $0,004)$, mientras que entre los hombres no se demostró un peor cumplimiento con alguno de los dos productos.

Analizando a los pacientes sin tener en cuenta la preparación empleada, no apreciamos asociación entre una mala tolerancia objetiva y un peor cumplimiento de las pautas de limpieza, aunque sí observamos una mayor presencia de vómitos entre los que no la completaron $(\mathrm{p}=$ $0,03)$. Por el contrario, sí se demostró un mayor riesgo de no finalizar la preparación por parte de aquellos pacientes que la toleraron subjetivamente mal $(\mathrm{p}<0,02)$.

\section{DISCUSIÓN}

En el presente estudio se ha demostrado que, en la población general, tanto la tolerancia subjetiva a la preparación del colon como la aparición de un conjunto de efectos adversos durante esta fueron similares tanto con PEG como con NaP. Estos datos concuerdan con la mayor parte de los estudios revisados, donde la tolerancia a la limpieza con $\mathrm{NaP}$ fue tan buena o mejor que con PEG $(1-4,6,8,12-14,17,18)$.

Analizando el grupo de pacientes de 65 años o más, que era el objetivo primario del estudio, tampoco se observaron diferencias de tolerancia subjetiva u objetiva en función del preparado, coincidiendo por tanto con los resultados de Thomson y cols. (16).

A pesar de ello, sí se apreció una relación entre la aparición de náuseas y la preparación con $\mathrm{NaP}$, debida al grupo de mujeres de 65 años o más. En el estudio de Frommer y cols. también se obtuvo una mayor frecuencia de náuseas con $\mathrm{NaP}$, pero no analizaron el papel que podían 
jugar edad o sexo (7). Asimismo, Seinelä y cols . confirman esta relación en un estudio con pacientes ingresados de 80 años o más, nuevamente sin estratificar por sexo (15). Las náuseas podrían estar causadas por irritación de la mucosa gástrica debido a la alta osmolaridad del producto (1.700 mOsm/l) (7). En el estudio de Bujanda y cols. se sugiere que este efecto podría atenuarse administrando un segundo vaso de líquido tras cada una de las dosis, ya que así disminuiría la osmolaridad de la solución (5). Por contra, no hemos observado una mayor frecuencia de vómitos o sed en los pacientes preparados con $\mathrm{NaP}$, hecho también descrito previamente (7).

En nuestro estudio no evidenciamos una asociación entre la mala tolerancia objetiva y uno cualquiera de los dos preparados en función del sexo. Sin embargo, observamos que las mujeres presentaron peor tolerancia subjetiva y una mayor frecuencia en la aparición de efectos adversos independientemente del producto empleado, lo cual, y según nuestro conocimiento, no se ha descrito previamente. Estas diferencias podrían justificarse por una menor tolerancia a la distensión gástrica aguda por líquidos en el sexo femenino, que se ve potenciada por una mayor prevalencia de la dispepsia funcional en este sexo (19), lo que reduce aún más el volumen de líquido ingerido sin que aparezcan náuseas u otra sintomatología gástrica (20). Esta menor capacidad para ingerir líquidos podría condicionar tanto una mayor dificultad para completar la preparación como un aumento de los síntomas objetivos y subjetivos a la misma. Otra posibilidad es que se deban al propio diseño retrospectivo del estudio, quizá por un mejor recuerdo del proceso de preparación por parte de las mujeres.

Resulta llamativo que los pacientes de 65 años o más toleraron objetiva y subjetivamente mejor la preparación si no se tiene en cuenta el producto empleado. Quizá uno de los factores implicados sea una alteración del umbral de la sensibilidad en este grupo de población, aunque es difícil de afirmar porque no hemos encontrado datos similares en la literatura revisada. De nuevo, el diseño retrospectivo podría influir en estos resultados, aunque es poco probable que los pacientes olvidaran mencionar algún efecto adverso, ya que en la entrevista fueron interrogados específicamente por cada uno de ellos.

Se han investigado otras dos variables que, al menos a nivel teórico, podrían influir en la tolerancia (subjetiva $u$ objetiva), como son la comorbilidad de los pacientes y los hallazgos endoscópicos, sin observar relación con esta tanto en el análisis uni- como en el multivariable. En el caso de la comorbilidad (cardio-, neumo- o hepatopatía), la escasa prevalencia entre los pacientes incluidos no permite realizar un estudio adecuado, mientras que en el de la patología orgánica colónica probablemente la muestra sea suficiente. Tampoco hemos encontrado referencias a estos factores en la literatura revisada.

Independientemente también del producto empleado, el cumplimiento de la pauta de limpieza no fue peor en aquellos pacientes que presentaron una mala tolerancia objeti- va, considerada como la presencia de al menos un efecto adverso, pero sí fue influido por la tolerancia subjetiva, lo cual sugiere que esta podría estar compuesta además de por los efectos adversos, por otros elementos de tipo funcional. Por otra parte, es razonable que los pacientes que presentaron vómitos no completaran la preparación en un mayor porcentaje que aquellos que no los tuvieron.

Sin embargo, teniendo en cuenta el preparado sí que se observó un mayor cumplimiento de la pauta con $\mathrm{NaP}$ a expensas del grupo de mujeres de 65 años o más, muy posiblemente debido a la menor ingesta de líquidos necesaria para completar la preparación $(19,20)$. Estos datos coinciden con los obtenidos en algunos estudios previos $(1,2,8,18,21)$, aunque en ellos no se establece relación con la edad o el sexo. La OR a favor del cumplimiento con $\mathrm{NaP}$ es bastante similar, por ejemplo, al riesgo relativo descrito en el meta-análisis de Hsu y cols. (2). Por el contrario, en el trabajo de Young y cols., donde la pauta de PEG implicaba la ingesta de sólo 2 litros de líquido, el grado de cumplimiento de esta era mucho mayor que en nuestro estudio (13).

Con respecto a la mayor calidad de la limpieza obtenida con NaP en nuestros pacientes, probablemente se deba a un mejor cumplimiento de la preparación, ya que teniendo en cuenta sólo a aquellos pacientes que la completaron no encontramos diferencias significativas entre ambos productos, a pesar de detectar una tendencia a favor del NaP. Lo que parece evidente es que dicha diferencia no se debe a los enemas, ya que estos fueron empleados con más frecuencia por los pacientes preparados con PEG; al ser conscientes de su peor preparación aumentaron su utilización, aunque sin alcanzar la significación estadística. En todo caso, no hay que olvidar que una mejor limpieza del colon aumenta la rentabilidad diagnóstica y reduce los costes al ahorrar nuevas colonoscopias $(2,12)$.

Otros autores también han demostrado que la limpieza del colon es mejor con $\mathrm{NaP}(5,8,12,13)$, observando algunos de ellos que la probabilidad de tener que repetir la colonoscopia por restos fecales es mayor en aquellos pacientes preparados con PEG $(1,2)$. Solamente en el estudio de Vanner y cols. (8) se sugiere una relación entre la peor limpieza con PEG y el no haber completado la preparación, mientras que en el resto no se analiza el papel que puede jugar el haber finalizado o no la preparación, o incluso se excluye del análisis a los pacientes que no completaron la pauta (4).

Se ha especulado sobre la importancia del momento de administración de ambos productos, concluyendo Frommer y cols. y otros grupos que la limpieza era mejor con $\mathrm{NaP}$ cuando la segunda dosis se administraba horas antes de la colonoscopia $(8,12)$, mientras que no había diferencias con el PEG si ambos productos se tomaban el día previo a la exploración $(7,13,17)$. Sin embargo, en otros estudios no se observaron diferencias entre PEG y NaP a pesar de emplear esta pauta (18). Destaca, por alcanzar conclusiones opuestas, el estudio de Ell y cols., donde se administraron las dos dosis de $\mathrm{NaP}$ el día previo a la colo- 
noscopia, y una segunda dosis de PEG en la mañana de la exploración, logrando una mejor preparación con PEG (3). El elevado porcentaje de limpieza buena o muy buena en dicho estudio ( $83 \%$ ) no concuerda con el equivalente en nuestro trabajo (47\%), posiblemente porque el cumplimiento de la pauta de PEG fue mucho mejor en esa población (3).

Aunque en el presente estudio no obtuvimos determinaciones bioquímicas, hemos demostrado previamente (22), de acuerdo con otros trabajos, que las alteraciones iónicas en pacientes sanos son más frecuentes entre aquellos preparados con $\mathrm{NaP}$ que con PEG, pero también que muy raras veces son clínicamente relevantes, incluso en población anciana $(2,3,6,9,12,15,16,18)$. De hecho, en nuestro estudio sólo una paciente (47 años) precisó ingreso hospitalario tras el proceso, y había realizado la preparación con PEG. A pesar de los potenciales efectos cardiacos secundarios a las alteraciones iónicas, hasta el momento no se ha observado una mayor frecuencia de arritmias cardiacas durante la preparación con cualquiera de los dos productos (18). Beloosesky y cols. concluyeron en pacientes de 65 años o más que la preparación con $\mathrm{NaP}$ producía alteraciones iónicas importantes (hiperfosfatemia, hipernatremia, hipocalcemia e hipopotasemia, fundamentalmente), pero transitorias y sin repercusión clínica. Sin embargo, no comparaban este grupo con otro de características similares preparado con PEG (9). En la literatura revisada hemos encontrado algunos casos de hipocalcemia sintomática tras preparación con $\mathrm{NaP}$, uno de ellos con resultado de muerte $(10,11)$. En todos ellos, o bien se produjo una sobredosificación del producto (oral o en forma de enemas), o bien este estaba contraindicado por presentar los pacientes insuficiencia renal crónica, o ambas cosas, lo cual apoya lo dicho hasta el momento en cuanto a su seguridad si es administrado a la dosis adecuada y en pacientes sin contraindicaciones.

En conclusión, la tolerancia subjetiva y objetiva a cualquiera de las dos preparaciones (PEG y NaP) fue similar en la población general y en los pacientes de 65 años o más, aunque los pacientes que se prepararon para la colonoscopia con $\mathrm{NaP}$ presentaron náuseas con mayor frecuencia que los que lo hicieron con PEG, fundamentalmente a expensas de las mujeres de 65 años o más. La tolerancia subjetiva y objetiva fue peor independientemente del producto en pacientes por debajo de 65 años y en mujeres. Los pacientes a los que se les administró NaP cumplieron mejor la pauta de limpieza que aquellos tratados con PEG, diferencia debida también al grupo de mujeres de 65 años o más, logrando una mejor limpieza del colon. Por tanto, la preparación con $\mathrm{NaP}$ es segura y eficaz también en pacientes ancianos sin patología asociada grave.

Sería conveniente realizar un estudio comparativo prospectivo y aleatorizado para confirmar estos resultados, algunos de los cuales no coinciden con los publicados en la literatura o, según nuestro conocimiento, no han sido estudiados previamente.

\section{NOTA}

No existe ningún conflicto de intereses por parte de los autores.

\section{AGRADECIMIENTOS}

Agradecemos a María Enciso su colaboración en este trabajo.

\section{BIBLIOGRAFÍA}

1. Curran MP, Plosker GL. Oral sodium phosphate solution. A review of its use as a colorectal cleanser. Drugs 2004; 64: 1697-714.

2. Hsu CW, Imperiale TF. Meta-analysis and cost comparison of polyethylene glycol lavage versus sodium phosphate for colonoscopy preparation. Gastrointest Endosc 1998; 48: 276-82.

3. Ell C, Fischbach W, Keller R, Dehe M, Mayer G, Schneider B, et al. A randomized, blinded, prospective trial to compare the safety and effectiveness of bowel-cleansing solutions. Endoscopy 2003; 35: 300-4.

4. Poon CM, Lee DW, Mak SK, Ko CW, Chan KC, Chan KW, et al. Two liters of polyethylene glycol-electrolyte lavage solution versus sodium phosphate as bowel cleansing regimen for colonoscopy: A prospective randomized controlled trial. Endoscopy 2002; 34: 560-3.

5. Bujanda L, Muñoz C, Sánchez A, Iriondo C, Ramosa F, Sánchez Llona $\mathrm{B}$, et al. Tolerancia y limpieza del colon con dos preparaciones. Polietilenglicol o fosfato sódico. Gastroenterol Hepatol 2001; 24: 9-12.

6. Nelson BD, Barkun AN, Block KP, Burdick JS, Ginsberg GG, Greenwald DA, et al. Technology status evaluation report. Colonoscopy preparations. May 2001. Gastrointest Endosc 2001; 54: 829-32.

7. Frommer D. Cleansing ability and tolerance of three bowel preparations for colonoscopy. Dis Colon Rectum 1997; 40: 100-4.

8. Vanner S, MacDonald P, Paterson W, Prentice R, Da Costa L, Beck I. A randomized prospective trial comparing oral sodium phosphate with standard polyethylene glycol-based lavage solution in the preparation of patients for colonoscopy. Am J Gastroenterol 1990; 85: 422-7.

9. Beloosesky Y, Grinblat J, Weiss A, Grosman B, Gafter U, Chagnac A. Electrolyte disorders following oral sodium phosphate administration for bowel cleansing in elderly patients. Arch Intern Med 2003; 163: 803-8

10. Boivin MA, Kahn SR. Symptomatic hypocalcemia from oral sodium phosphate: A report of two cases. Am J Gastroenterol 1998; 93: 2577-9.

11. Knobel B, Petchenko P. Hyperphosphatemic hypocalcemic coma caused by hypertonic sodium phosphate (fleet) enema intoxication. J Clin Gastroenterol 1996; 23: 217-9.

12. Kolts BE, Lyles WE, Achem SR, Burton L, Geller AJ, MacMath T. A comparison of the effectiveness and patient tolerance of oral sodium phosphate, castor oil, and standard electrolyte lavage for colonoscopy or sigmoidoscopy preparation. Am J Gastroenterol 1993; 88: 1218-23.

13. Young CJ, Simpson RR, King DW, Lubowski DZ. Oral sodium phosphate solution is a superior colonoscopy preparation to polyethylene glycol with bisacodyl. Dis Colon Rectum 2000; 43: 1568-71.

14. Pou Fernández JM, Rodríguez Muñoz S, Sala Felis T. Caracterización del perfil de seguridad, eficacia y utilización de fosfato sódico oral. Rev Esp Enferm Dig 2001; 93: 214-9.

15. Seinelä L, Pehkonen E, Laasanen T, Ahvenainen J. Bowel preparation for colonoscopy in very old patients: A randomized prospective trial comparing oral sodium phosphate and polyethylene glycol. Scand J Gastroenterol 2003; 38: 216-20.

16. Thomson A, Naidoo P, Crotty B. Bowel preparation for colonoscopy: A randomized prospective trail comparing sodium phosphate and polyethylene glycol in a predominantly elderly population. J Gastroenterol Hepatol 1996; 11: 103-7.

17. Henderson JM, Barnett JL, Turgeon DK, Elta GH, Behler EM, Crause I, et al. Single-day, divided-dose oral sodium phosphate laxative versus intestinal lavage as preparation for colonoscopy: Efficacy and patient tolerance. Gastrointest Endosc 1995; 42: 238-43. 
18. Clarkston WK, Tsen TN, Dies DF, Schratz CL, Vaswani SK, Bjerregaard P. Oral sodium phosphate versus sulfate-free polyethylene glycol electrolyte lavage solution in outpatient preparation for colonoscopy: A prospective comparison. Gastrointest Endosc 1996; 43: 42-8

19. Shaib Y, El-Serag HB. The prevalence and risk factors of functional dyspepsia in a multiethnic population in the United States. Am J Gastroenterol 2004; 99: 2210-6.

20. Jones MP, Hoffman S, Shah D, Patel K, Ebert CC. The water load test: Observations from healthy controls and patients with functional dys- pepsia. Am J Physiol Gastrointest Liver Physiol 2003; 284: 896-904.

21. Afridi S, Barthel J, King P, Pineda J, Marshal J. Prospective, randomized trial comparing a new sodium phosphate-bisacodyl regimen with conventional PEG-ES lavage for outpatient colonoscopy preparation. Gastrointest Endosc 1995; 41: 485-9.

22. Marín Gabriel JC, Rodríguez Muñoz S, De la Cruz Bértolo J, Carretero Gómez F, Muñoz Yagüe MT, Manzano Alonso ML, et al. Alteraciones electrolíticas y colonoscopia: preparación, edad y procedimiento. Rev Esp Enferm Dig 2003; 95: 863-9. 